\title{
Estudio Comparativo del Estrés en las Docentes de las Instituciones Educativas de Educación Inicial en el Perú
}

\author{
Comparative Study of Stress in Teachers Educational Institutions Early Education in the \\ Peruvian
}

\begin{abstract}
Norma Adela Moreno Rivera', Perfecta Zobeida Fernández Guzmán², Maritza Isabel Martínez Loli', Julia María Yábar Rayo², Olga Marina Gamarra Lopez ${ }^{1}$, Karen Sánchez Cóndor ${ }^{2}$, Bethsabeth Melosich Aviles ${ }^{1}$, Luz Mery Tadeo Sifuentes ${ }^{1}$
\end{abstract}

\section{RESUMEN}

Objetivo: Comparar el nivel de estrés en las docentes de las instituciones educativas de educación Inicial en el Perú. Métodos: Fue una investigación descriptiva transversal. La población estuvo formada por 89 docentes de las instituciones públicas y 92 de las instituciones privadas. La muestra fue de tipo no probabilístico. Para la recolección de datos se empleó una encuesta o ficha de datos personales y el test de Maslach constituido por 22 ítems con 6 alternativas de respuesta. Se evaluó tres dimensiones: Agotamiento emocional, despersonalización y realización personal. Para el procesamiento de la información se utilizó el programa estadístico SPSS versión 20. Resultados: Se obtuvo un Alfa de Cronbach de 0,627 y de 0,753 para los datos de los docentes de Centros educativos privado y Centros educativos estatales respectivamente. La edad promedio de las docentes de gestión pública y privada son de 41,77 y 30,12 años respectivamente; el tiempo de servicios son de 11,68 y 3,36 años, respectivamente. Se aplicó la Prueba de U de Mann-Withney para muestras independientes y se demostró que existe diferencia significativa en las edades y tiempo de servicio de las docentes de ambos grupos. Hay diferencias significativas en el nivel de estrés de los docentes del nivel inicial que laboran en instituciones de gestión pública y privada. Conclusión: Las docentes que laboran en las instituciones educativas de gestión privada presentaron mayor puntuación de estrés que las docentes que laboran en las instituciones educativas de gestión pública a pesar de que éstos últimos son de mayor edad y de mayor tiempo de servicio.

Palabras clave: Estrés, agotamiento emocional, despersonalización, realización personal, gestión pública, gestión privada.

\section{ABSTRACT}

Objective: To compare different levels of stress on teachers in early education institutions in Peru. Methods: It was a descriptive investigation. The population consisted of 89 teachers in public institutions and 92 private institutions. The sample was not probabilistic. Data collection for a survey or record of personal data and the Maslach test consists of 22 items with 6 possible answers was used. Emotional exhaustion, depersonalization and personal accomplishment: three dimensions are evaluated. Cronbach Alfa of 0.627 and 0.753 were obtained for data CEP teachers and EEC respectively for information processing SPSS version 20. Results: are used. The average age of teachers in Public and Private Management are 41.77 and 30.12 years respectively; time services are 11.68 and 3.36 years, respectively. Test Mann-Whitney was applied for independent samples and showed that there are significant differences in age and length of service of teachers in both groups. There are significant differences in the stress level of the initial level teachers who work in institutions of Public and Private Management. Conclusion: The teachers who work in educational institutions Private Management showed higher stress scores than teachers who work in educational institutions of governance even though the latter are older and longer service life.

Keywords: Stress, emotional exhaustion, depersonalization, personal accomplishment, public management, private management.

\footnotetext{
${ }^{1}$ Facultad de Ciencias Sociales. Universidad Nacional José Faustino Sánchez Carrión. Huacho, Perú.

${ }^{2}$ Facultad de Educación. Universidad Nacional José Faustino Sánchez Carrión. Huacho, Perú.
} 


\section{INTRODUCCIÓN}

En la psicología, sociología y pedagogía se ha desarrollado un gran interés por estudiar el estrés y los factores psicosociales relacionados con el trabajo y su repercusión en la salud de los trabajadores y en los resultados en el trabajo. Lazarus y Folkman (1986) señalan que el estrés es un conjunto de relaciones particulares entre las personas y la situación, siendo valorada por la persona como algo grave o que excede sus propios recursos que pone en peligro su bienestar personal.

El trabajo cotidiano de las docentes de educación inicial implica no solo la parte educativa sino también la responsabilidad de la atención de su alimentación, higiene, salud, velar por la integridad física y mental teniendo en cuenta que el niño se encuentra en el periodo más crítico del desarrollo, donde se sientan las bases de la personalidad y más aún en esta etapa ocurre el desarrollo hasta de un $70 \%$ de la inteligencia. Lo que implica, que esta responsabilidad que tienen las docentes de educación inicial significa actuar siempre bajo presión laboral.

Guerrero y Rubio (2005) señalan que el burnout es un término Inglés que significa literalmente "estar quemado por el trabajo", cuyo término décadas atrás; fue difundido y consolidado por Maslach y Jackson (1981) quienes consideraron que el burnout era un síndrome tridimensional derivado de un estrés emocional crónico cuyos rasgos principales son cansancio físico y/o psicológico, junto con la sensación de no poder dar más de sí mismo (agotamiento emocional), una actitud fría y despersonalizada en relación con los demás (despersonalización) y un sentimiento de inadecuación al puesto de trabajo y a las tareas que el afectado desarrolla con bajo logro personal y profesional. Asimismo Guerrero (1999) señala que desde el enfoque psicosocial, el síndrome de Burnout no debe ser considerado solo como estrés psicológico; sino entenderse como una respuesta a las fuentes de estrés crónico desarrollados a partir de las relaciones entre los usuarios de servicios de asistencia (pacientes, alumnos, clientes, etc.) y los profesionales que los atienden (médicos, docentes, policías, abogados, etc.) es decir su revisión y análisis sobre este problema también se presenta entre los profesionales de la educación.
Autores como: Kyriacou, 1980; Cunningham, 1983; Ivancevich, 1988; Pierce, Molloy y Ogus, 1992; Moreno, Oliver y Aragoneses, 1992; Manassero, Vázquez, Ferrer, Fornés, Fernández y Queimaledos, 1994; Oliver 1993; Salgado, Yela, Quevedo, Delgado, Fuentes, Sánchez y Velasco, 1997; Aluja, 1997; Guerrero, 1999 y Rubio, 2003; citado por Guerrero y Rubio (2005) han coincidido en señalar que se puede identificar el síndrome de burnout en los profesionales de la educación debido a que tienen que enfrentarse con problemas somáticos y psicológicos que dañan significativamente la labor profesional, su relación con los estudiantes y calidad de la enseñanza.

Atalaya, (2001) identificó que la solución del problema del estrés debe involucrar a la organización y al trabajador como actores principales del tema; es decir, habrá mayor eficacia en las estrategias que estén dirigidas a disminuirlo o solucionarlo si hay unión de esfuerzos de un lado los conductores de las organizaciones quienes con responsabilidad deben asumir su papel que le corresponde porque la mayor fuentes de estrés provienen de este espacio y además de los trabajadores y profesionales que laboran quienes deben entender que vivimos en una época de cambio y de conflictos permanentes, aprender a enfrentar nuevos retos y exigencias pero que al lograrlos tendrá más sentido la convivencia y la satisfacción personal.

Ponce, Bulnes, Aliaga y Atalaya (2005) tienen como objetivos: conocer la posible presencia del Síndrome del "quemado" por estrés laboral en grupos de docentes de diversas áreas. Describir posibles diferencias significativas en el síndrome del "quemado" por estrés laboral. Como conclusiones obtuvieron que los docentes de la UNMSM presentan evidencias del síndrome del "quemado" por estrés laboral asistencial. El análisis cualitativo permitió apreciar diferencias en la presencia de "Burnout" según áreas de especialidad. No existen diferencias en las etapas de "Burnout" en relación a las variables de sexo, estado civil. Existen diferencias significativas en las etapas de "Burnout" en relación a las variables: Problemas de salud y condición docente y concluyen que hubo mayor cansancio emocional y alto nivel de despersonalización. 
Moreno, Fernández, Martínez, Yábar y Vidaurre (2013) realizaron un estudio del nivel de estrés de los docentes de las Facultades de Educación y Ciencias Sociales de la Universidad Nacional José Faustino Sánchez Carrión, a fin de comparar el nivel de estrés de docentes en ambas facultades. La muestra no probabilística estuvo constituida por el $27,33 \%$ y $30,76 \%$ respectivamente. Se aplicó el test de Maslash cuya conclusión fue: Los docentes de ambas facultades presentaron estrés promedio equivalente a estrés agudo, el $90,24 \%$ y $80,0 \%$ presentan niveles Bajos de agotamiento, el $61 \%$ y $55 \%$ tienen bajos niveles de despersonalización y el $56 \%$ y $40 \%$ presentan altos niveles de realización personal. Teniendo en cuenta la importancia de la etapa formativa inicial en lo social y cognitiva y motora de los infantes, el docente responsable debe ser saludable emocionalmente y libre de estrés que asegure su desempeño profesional.

Por lo que como hipótesis nos planteamos que en un ambiente agradable, sin presión laboral favorece el estado emocional de los docentes de las instituciones educativas de nivel inicial. El objetivo fue comparar el nivel de estrés en las docentes de las instituciones educativas del nivel inicial en el Perú.

\section{MATERIAL Y MÉTODOS}

Se realizó una investigación descriptiva comparativa; cuyo objetivo fue recolectar información relevante en dos muestras respecto a un mismo fenómeno o aspecto de interés y luego caracterizar este fenómeno en base a la comparación de los datos recogidos.

La población estuvo formada por 181 docentes de las instituciones educativas de educación inicial del distrito de Huacho, de los cuales 89 pertenecen a las instituciones de educación de gestión pública y 92 a las instituciones de gestión privada. Se trabajó con el muestreo estratificado no probabilístico formado por el $74,16 \%$ de docentes de las instituciones de educación inicial pública y el $45,65 \%$ de docentes de las instituciones de educación inicial privadas de Huacho. Para la obtención de datos referentes a la unidad de análisis, se utilizó una ficha de datos personales y el Test de Maslach que comprende tres dimensiones: Agotamiento o cansancio emocional, despersonalización y realización personal y consta de 22 ítems cuya categorización se detalla en la Tabla 1.

Tabla 1. Categorización del Test de Maslach

\begin{tabular}{llcl}
\hline \multirow{2}{*}{ Dimensiones } & \multicolumn{1}{c}{ Categorías } & Alta \\
\cline { 2 - 3 } & Baja & Media & 27 años \\
\hline Agotamiento emocional & 0 a 18 & 19 a 26 & 10 a más \\
Despersonalización & 0 a 05 & 06 a 09 & 40 a más \\
Realización personal & 0 a 30 & 31 a 39 & 67 a 99 \\
& 0 a 33 & 34 a 66 & \\
\hline
\end{tabular}

Fuente: Buzzeti, (2005)

Asimismo, para el procesamiento de datos se utilizó el SPSS versión 20 y para la prueba de las hipótesis, investigación que se planteó como hipótesis la confirmación si existe o no diferencias significativas en la edad, tiempo de servicio y el nivel de estrés de las docentes del nivel inicial que laboran en la instituciones educativas de gestión pública y privada arribándose a las conclusiones que finaliza la investigación realizada, por lo que se aplicó el test de $U$ de Mann Whitney, a un 95\% de confiabilidad.

\section{RESULTADOS}

Para conocer la fiabilidad del Test de Maslach, se aplicó la Prueba de Alfa de Cronbach que arrojó 0,753 para los centros educativos de gestión pública y 0,727 para los centros de educativos de gestión privada.

La edad promedio y la variación de las edades de los docentes que laboran en centros educativos de gestión pública, fue de 41,77 y los de Centros educativos de gestión privada de 30,12 años. 
Con respecto al tiempo de servicio de las docentes, se demostró que el tiempo de servicio promedio y la variación son mayores en las docentes que laboran en centros educativos de gestión pública $(11,68$ años) frente a los docentes de centros educativos de gestión privada ( 3,36 años).
Las estadísticas obtenidas de la aplicación del Inventario de Maslach, muestran los puntajes promedios de 64,76 y 71,07 para las docentes que laboran en centros educativos de gestión pública y privada, respectivamente. Hay mayor variación de datos, es decir; estos se encuentran más dispersos que en las docentes de centros educativos de gestión pública.

Tabla 2. Estadísticas de la aplicación del Inventario de Maslach

\begin{tabular}{lcc}
\hline $\begin{array}{c}\text { Puntajes } \\
\text { obtenidos }\end{array}$ & $\begin{array}{c}\text { Centros educativos } \\
\text { de Gestión Pública }\end{array}$ & $\begin{array}{c}\text { Centros educativos } \\
\text { de Gestión Privada }\end{array}$ \\
\hline & & 42 \\
Media & 66 & 71,07 \\
Mediana & 64,76 & 70,00 \\
Moda & 65,50 & $66 a$ \\
Desv. típ. & $58 a$ & 12,671 \\
Varianza & 13,619 & 160,556 \\
Asimetría & 185,479 & 0,232 \\
Curtosis & $-0,059$ & 0,189 \\
Rango & $-0,139$ & 55 \\
Mínimo & 62 & 44 \\
Máximo & 30 & 99 \\
a. Existen varias modas. Se mostrará el menor de los valores & 92 \\
\hline
\end{tabular}

En la Tabla 3 se comparan los puntajes obtenidos por niveles de estrés. En docentes que laboran en instituciones educativas de gestión pública y privada se observa un nivel de estrés Alto en un $54,63 \%$, y un nivel de estrés Medio con un $44,44 \%$. Por ultimo tenemos que un $0,93 \%$ tienen niveles bajos de estrés.

Tabla 3. Calificación de Niveles de estrés en las docentes de centros educativos públicos y privados

\begin{tabular}{|c|c|c|c|c|c|c|}
\hline \multicolumn{7}{|c|}{ Síndrome de Stress de Burnout } \\
\hline \multirow[t]{2}{*}{$\begin{array}{l}\text { Niveles de } \\
\text { estrés }\end{array}$} & \multicolumn{2}{|c|}{$\begin{array}{l}\text { Instituciones educativas } \\
\text { públicas }\end{array}$} & \multicolumn{2}{|c|}{$\begin{array}{c}\text { Instituciones educativas } \\
\text { privadas }\end{array}$} & \multicolumn{2}{|c|}{ Total } \\
\hline & $\mathbf{F i}$ & $\%$ & $\mathbf{F i}$ & $\%$ & $\mathbf{n}$ & $\%$ \\
\hline Alto & 30 & 45,5 & 29 & 69,0 & 59 & 54,63 \\
\hline Medio & 35 & 53,0 & 13 & 31,0 & 48 & 44,44 \\
\hline Bajo & 1 & 1,5 & 0 & 0 & 1 & 0,93 \\
\hline TOTAL & 66 & 100 & 42 & 100 & 108 & 100,00 \\
\hline
\end{tabular}

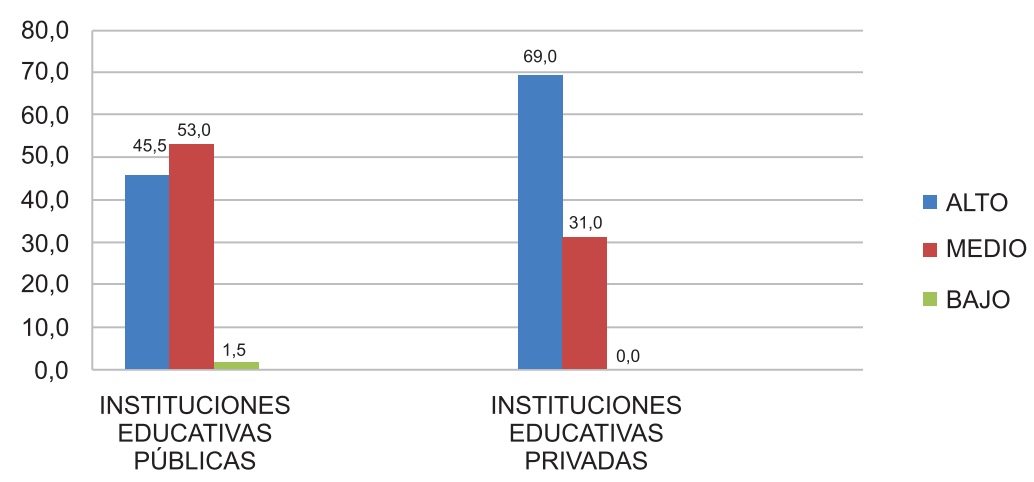

Figura 1. Niveles de estrés de las docentes de centros educativos públicos y privados 
En la dimensión de Agotamiento, las puntuaciones altas están en el nivel bajo en los docentes que laboran en las instituciones de gestión pública a diferencia de las puntuaciones de nivel medio en docentes que laboran en instituciones educativas de gestión privada. Figura 2.

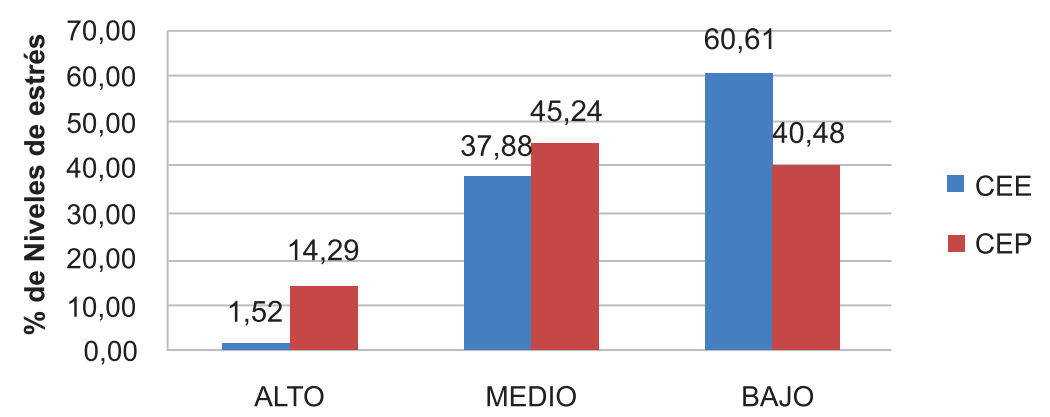

Figura 2. Niveles de la dimensión Agotamiento en las docentes de centros educativos públicos y privados

En la dimensión Despersonalización, un $80,30 \%$ y $57,14 \%$ de los docentes que laboran en instituciones educativas de gestión pública y de gestión privada, respectivamente; tienen mayores puntuaciones en el nivel bajo. Solo el
$7,14 \%$ de los docentes que laboran en instituciones educativas de gestión privada tienen un nivel de estrés alto, mientras que esta dimensión no se presenta en los docentes de instituciones de gestión pública, (Figura 3).

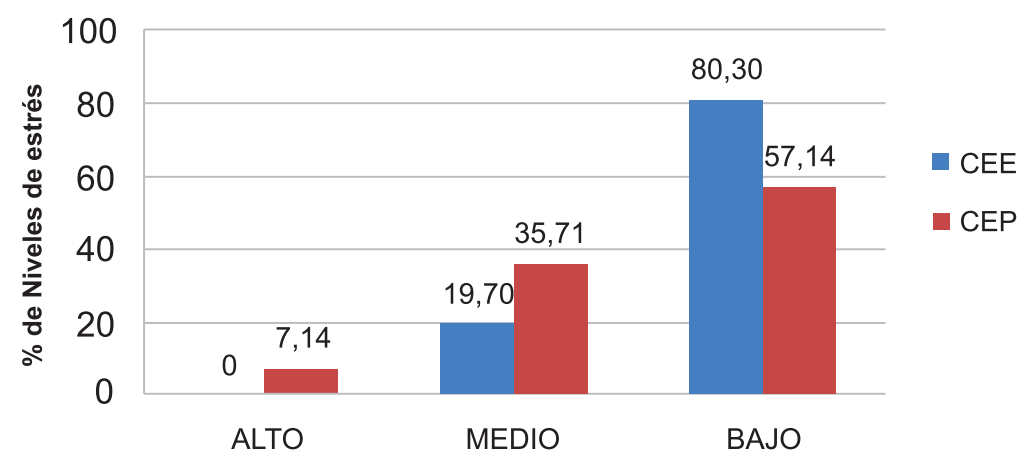

Figura 3. Niveles de la dimensión de Despersonalización en las docentes de centros educativos públicos y privados

En la dimensión de Realización Personal, las mayores puntuaciones se ubican en el nivel alto de Realización Personal para las docentes que laboran en ambas instituciones educativas, (Figura 4).

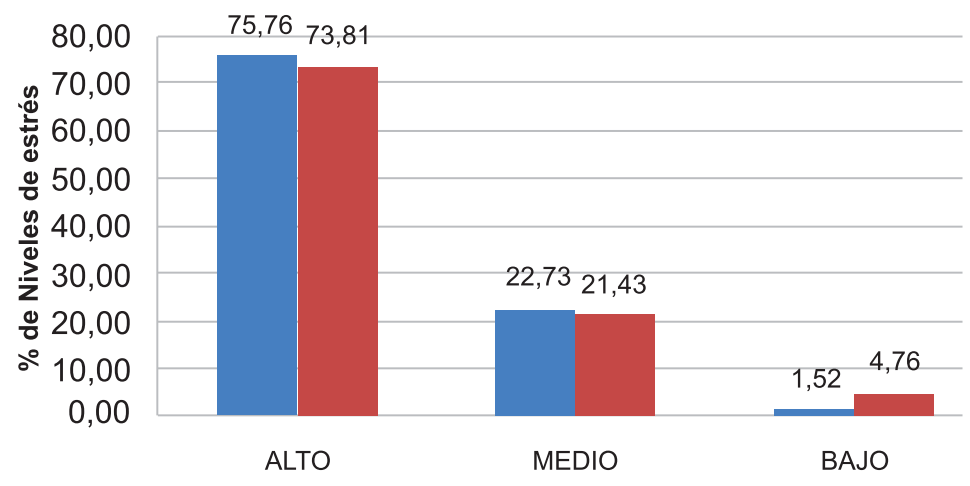

Figura 4. Niveles de la dimensión de Realización Personal en las docentes de centros educativos públicos y privados 
Se docimó las hipótesis aplicando la Prueba de U de Mann Whitney, a un 95\% de confiabilidad.

Al docimar la primera hipótesis específica, se obtuvo un Sig. Bilateral $=0,000$ lo que nos demuestra que existe diferencia significativa en la edad de las docentes de ambos grupos, siendo de mayor edad las docentes de instituciones educativas de gestión pública.

\section{Resumen de prueba de hipótesis}

\begin{tabular}{|c|c|c|c|c|}
\hline & Hipótesis nula & Test & Sig. & Decisión \\
\hline 1 & $\begin{array}{l}\text { La distribución de EDAD es la } \\
\text { misma entre las categorías de TIPO } \\
\text { DE GESTIÓN DEL CENTRO } \\
\text { EDUCATIVO }\end{array}$ & $\begin{array}{l}\text { Prueba U de } \\
\text { Mann-Whitney } \\
\text { de muestras } \\
\text { independientes }\end{array}$ & ,000 & $\begin{array}{l}\text { Rechazar la } \\
\text { hipótesis nula }\end{array}$ \\
\hline
\end{tabular}

Se muestran las significancias sintóticas. El nivel de significancia es ,05.

Al docimar la segunda hipótesis específica, se obtuvo un Sig. Bilateral $=0,000$ lo que nos demuestran que existe diferencia significativa en el tiempo de servicio de las docentes de ambos grupos, siendo de mayor tiempo de servicio las docentes de instituciones educativas de gestión pública.

Resumen de prueba de hipótesis

\begin{tabular}{|c|c|c|c|c|}
\hline & Hipótesis nula & Test & Sig. & Decisión \\
\hline 1 & $\begin{array}{l}\text { La distribución de TIEMPO DE } \\
\text { SERVICIO es la misma entre las } \\
\text { categorías de TIPO DE GESTIÓN } \\
\text { DEL CENTRO EDUCATIVO }\end{array}$ & $\begin{array}{l}\text { Prueba U de } \\
\text { Mann-Whitney } \\
\text { de muestras } \\
\text { independientes }\end{array}$ &, 000 & $\begin{array}{l}\text { Rechazar la } \\
\text { hipótesis nula }\end{array}$ \\
\hline
\end{tabular}

Se muestran las significancias sintóticas. El nivel de significancia es ,05.

Al docimar la hipótesis general, se obtuvo un Sig. Bilateral $=0,018 \mathrm{lo}$ cual nos indica que existe diferencia significativa en los niveles de estrés en las docentes que laboran en las instituciones educativas de gestión pública y privada, siendo las docentes de instituciones privadas las de mayor nivel de estrés.

\section{Resumen de prueba de hipótesis}

\begin{tabular}{lllll}
\hline Hipótesis nula & Test & Sig. & Decisión \\
\hline La distribución de PUNTAJE & $\begin{array}{l}\text { ACUMULADO es la misma entre las } \\
\text { categorías de TIPO DE GESTIÓN } \\
\text { DEL CENTRO EDUCATIVO }\end{array}$ & $\begin{array}{l}\text { Prueba U de } \\
\text { Mann-Whitney } \\
\text { de muestras } \\
\text { independientes }\end{array}$ & ,018 & $\begin{array}{l}\text { Rechazar la } \\
\text { hipótesis nula }\end{array}$ \\
\hline
\end{tabular}

Se muestran las significancias sintóticas. El nivel de significancia es ,05.

\section{DISCUSIÓN}

El estudio arrojo que la edad promedio de 42 y 30 años para los docentes de instituciones públicas y privadas, respectivamente. Esta diferencia es significativa. Así, siguiendo a la teoría psicosocial de Erickson (2000), concluimos que los docentes de las instituciones de gestión públicas y privadas están en promedio en la edad adulta media, que es una etapa denominada Generatividad frente al aislamiento, que dura desde los 25 hasta los 60 años aproximadamente, donde se establece la carrera asumida, una relación emocional, se inicia la formación de la propia familia y desarrolla una sensación de ser parte de algo más amplio. Se aporta algo a la sociedad al criar a nuestros hijos, ser 
productivos en el trabajo, y participar en las actividades y organización de la comunidad. Si no alcanzamos estos objetivos, nos quedamos estancados y con la sensación de no ser productivos.

El tiempo de servicio promedio es de 11,68 y 3,36 años para los docentes de instituciones públicas y privadas, respectivamente. Esta diferencia es significativa, no obstante el nivel de estrés se presenta más alto en los docentes de las instituciones de gestión privada. Parada et al., (2005) en su estudio revelan la conformación de un perfil epidemiológico del personal de enfermería en un Hospital Universitario, caracterizado por: menores de 40 años, con menos de 20 años de servicio en la institución, con pareja habitual y con más de $60 \%$ de tiempo laboral diario en contacto directo con el paciente, lo que refleja la instauración de esta patología (ESTRÉS) en personas cada vez más jóvenes, con pocos años en el mundo laboral y con un tiempo mayor de dedicación al paciente. Por otro lado, Moriana y Herruzo, (2004) en su investigación identificó algunas variables en relación al estrés tal es así que sobre edad los resultados son contradictorios puesto que la relación establecida entre la edad y el burnout ha sido lineal una asociación que demuestra una relación directa de ambas variables, el autor cita a Seltzer y Numerof, (1988) y a Hock (1988) que encontró ausencia de relación en su investigación. Es decir, los resultados son diferentes sin embargo si a este análisis de síndrome de burnout y la edad, se asocia el tiempo de experiencia en la profesión, la maduración propia por la edad del individuo y con la pérdida de una visión irreal de la vida en general los resultados son más exactos. Es así que los profesores más jóvenes experimentan niveles superiores de estrés (Yagil, 1998) y mayores niveles de cansancio emocional y fatiga (Crane e Iwanicki, 1986; Schawb e Iwanicki, 1982a) citados por Moriana y Herruzo (2004). Asimismo, los autores mencionados señalan que en las investigaciones de Van Ginkel (1987) y Borg y Falzon (1989) informaban que los docentes más experimentados, que llevaban más de veinte años en la profesión, tenían una respuesta de estrés mayor que la de sus compañeros.

Los resultados del estudio comparativo demuestran que los docentes de las instituciones públicas tienen nivel de estrés medio, mientras que los docentes de instituciones privadas tienen nivel de estrés alto. Tales resultados nos indican que podríamos estar frente a un problema psicológico individual de origen social, que genera un conjunto de síntomas como: falta de ilusión y expectativas, apatía, ansiedad y depresión, agotamiento físico y mental, entre otros factores que tienen incidencia en la salud física y mental del docente que traen como consecuencia las bajas laborales, sentimiento de desconcierto e insatisfacción, inhibiciones, petición de traslados, abandono de la docencia, agotamiento, ansiedad, depresión y el absentismo; todos ellos relacionados directamente con la existencia del estrés acumulado a lo largo de la labor docente. "El estrés laboral, se ha convertido en una de las principales causas de baja en algunas profesiones, entre las que se encuentra la docencia." Pérez (2012).

Haciendo comparaciones con las subdimensiones, tenemos que los docentes de las instituciones educativas públicas presentan bajos niveles de agotamiento emocional y los docentes de las instituciones educativas privadas presentan niveles medios y bajos de estrés; es decir, una mínima sensación de estar exhaustos emocionalmente por las demandas del trabajo académico. Asimismo, en la dimensión despersonalización, tenemos que ambos grupos de docentes poseen niveles considerables de estrés, porque al menos cada uno reconoce actitudes de frialdad, distanciamiento y falta de sentimientos hacia los sujetos, sin embargo; necesitan aprender estrategias adecuadas de afrontamiento. Por último, los docentes de ambas grupos tienen un alto nivel en la sub dimensión de realización personal, que se caracteriza por una buena percepción en sus capacidades de autoeficacia y autoeficiencia en su labor y fuertes sentimientos de competencia y realización exitosa en el trabajo hacia los demás.

Los resultados de Restrepo, Colorado y Cabrera (2005) en la investigación desarrollada en 239 docentes de Medellín, concluyeron que el 23,4\% presentó manifestaciones de Burnout y un $23,4 \%$ adicional tuvo riesgo de manifestarlo; en ambos subgrupos prevaleció el agotamiento emocional y la despersonalización. Concluyeron que existe relación de manifestaciones del Burnout con algunas variables estudiadas; dichos 
resultados son parecidos a los de nuestro estudio: donde encontramos docentes de las instituciones educativas de gestión privada con niveles altos de estrés, probablemente expuestos a mayores horas de carga laboral, exigencia de cumplimiento de las labores asignadas con los infantes a su cargo e inestabilidad laboral que son sujetas; a diferencia de los docentes de las instituciones educativas de gestión pública quienes poseen un nivel medio de estrés, posiblemente porque saben controlar su estrés, su carga horaria es menor y la mínima exposición a estresores externos: transporte, inseguridad, tiempo prolongado de los docentes fuera del hogar, etc. que puede ser motivo de otra investigación. Aris (2009) en su investigación comprobó la existencia de un porcentaje elevado de maestros que podrían hacer Burnout, los resultados confirmaron que estaban ante ese importante problema que día a día crece. Concluyeron que algunos profesionales se ven forzados a implicarse por muchas horas en los problemas y preocupaciones de las personas con las que realiza su actividad laboral. Este es el caso de los maestros y docentes en general, cuya relación profesional se centra en el niño o adolescente $y$, concretamente en sus problemas, dificultades e inquietudes. Esta investigación se contrasta con la nuestra.

Salas (2010) en su investigación llegó a la conclusión: El Síndrome de Burnout en dichos docentes se presenta en un nivel alto, hay que resaltar que no encontró asociación significativa entre el síndrome de Burnout entre los docentes según el tipo de colegio llegando a la conclusión que el Síndrome de Burnout se puede dar tanto en colegios públicos y privados; a diferencia de los resultados de nuestro estudio donde se encontraron diferencias significativas entre ambos tipos de instituciones educativas.

Fernández-Arata, (2008), investigó en 929 profesores (617 mujeres y 312 varones) de escuelas primarias y secundarias de Lima, Perú. Los resultados muestran la existencia de un elevado nivel de Burnout en los profesores, siendo las puntuaciones más altas en docentes del nivel primario que en los de secundaria similar a nuestra investigación. Se encontró una significativa relación entre los estresores, conducta tipo A, satisfacción en el trabajo y variables de desempeño docente, tales como la auto-eficacia percibida, que puede verse como un factor protector contra el Burnout.

\section{AGRADECIMIENTO}

Al Director de la UGEL $N^{\circ} 09$ que autorizó la realización de la investigación

A todos aquellas docentes de las instituciones educativas públicas y privadas del nivel inicial que accedieron a la aplicación del test y que permitió arribar a los resultados antes mencionados.

\section{REFERENCIAS BIBLIOGRÁFICAS}

Aris, N. (2009). El Síndrome de Burnout en los docentes. Electronic Journal of Research in Educational Psychology. Universidad de Almeira. España,7(18), 829 - 848 . O bte n ido de: http://www.redalyc.org/pdf/2931/29312 1945012.pdf

Atalaya, M. (2001). El estrés laboral y su influencia en el trabajo. Industrial Data, $4(2), 25-36$.

Fernández-Arata, J. M. (2008). Desempeño docente y su relación con orientación a la meta, estrategias de aprendizaje y autoeficacia: un estudio con maestros de primaria de Lima, Perú. Universitas Psychologica, 7(2), 385-401.

Guerrero, E. (1999). Burnout o desgaste psíquico y afrontamiento del estrés en el profesorado universitario. Madrid: Premios Nacionales de Investigación Educativa, Ministerio de Educación, Ciencia y Cultura. 298-317.

Guerrero, E. \& Rubio, J. (2005). Estrategias de prevención e intervención del "burnout" en el ámbito educativo. Salud Mental, 28(5), pp. 27-33 Obtenido de http://www.medigraphic.com/pdfs/salm en/sam-2005/sam055d.pdf.

Lazarus, R. \& Folkman, S. (1986). Estrés y proceso Cognitivo. Barcelona: Martínez Roca.

Moreno, N., Fernández, S., Martínez, M., Yábar, J. \& Vidaurre, M. (2013). Estudio comparativo del nivel de estrés de los docentes de las Facultades de Educación y Ciencias Sociales de la Universidad Nacional José Faustino Sánchez Carrión. Infinitum..., 4(1), 6168. 
Moriana, J. \& Herruzo, J. (2004). Estrés y burnout en profesores. Universidad de Córdoba, España. (c) International Journal of Clinical and Health Psychology, 4(3), pp. 597-621. Obtenido en:

http://www.aepc.es/ijchp/articulos_pdf/ij chp-126.pdf

Parada, M. E., Moreno, B., Mejías, M., Rivas, F., Cerrada, S., \& Rivas, P. (2005). Satisfacción laboral y síndrome de burnout en el personal de enfermería del Instituto Autónomo Hospital Universitario Los Andes (IAHULA), Mérida, Venezuela, 2005. Revista Facultad Nacional de Salud Pública, 23(1), 33-45.

Pérez, P. (2012). El malestar docente. Temas para la Educación. Revista digital para Profesionales de la Enseñanza. Federación de la enseñanza de CC.OO de Andalucía. 21, (1), pp. 1-6

Ponce, C., Bulnes, M., Aliaga, J. \& Atalaya, M. (2005). El síndrome del "quemado" por estrés laboral Asistencial en grupos de Docentes Universitarios. IPSI, 8(2), pp. $87-112$. O b t e n i d o e n : http://sisbib.unmsm.edu.pe/BVRevistas /Investigacion_Psicologia/v08_n2/PDF/ a07.pdf
Restrepo, N., Colorado, G. \& Cabrera, G. (2005). Desgaste Emocional en Docentes Oficiales de Medellín, Colombia. Salud Pública, 8(1), pp. 6373.

Salas, J. (2010). Bienestar psicológico y síndrome de Burnout en docentes de la UGEL $N^{\circ} .7$. Tesis para optar el grado académico de Magíster en Psicología con Mención en Psicología Clínica y de la Salud, Lima: Universidad Nacional Mayor de San Marcos.

\section{Correo electrónico:}

normadelamoreno@hotmail.com

Revisado por Pares:

Recibido: 18-02-2015

Aceptado: 23-06-2015 